\title{
OPTIMAL CHAOTIC SELECTORS
}

\author{
ABRAHAM BOYARSKY, PAWEŁ GÓRA, AND ZHENYANG LI
}

\begin{abstract}
Multivalued maps have many applications. We consider one dimensional multivalued maps whose graphs are defined by lower and upper boundary maps. Let $I=[0,1]$ and let $\mathcal{P}$ be a partition of $I$ into a finite number of intervals. Let $\tau_{\ell}, \tau_{u}: I \rightarrow I$ be two piecewise expanding maps on $\mathcal{P}$ such that $\tau_{\ell} \leq \tau_{u}$. Let $G \subset I \times I$ be the region bounded by the graphs of $\tau_{\ell}$ and $\tau_{u}$. Any map $\boldsymbol{\eta}: I \rightarrow I$ that takes values in $G$ is called a selector of the multivalued map defined by $G$. We assume that $\tau_{\ell}$ and $\tau_{u}$ as well as all the selectors we consider have invariant distribution functions. Let $F^{*}$ be a target distribution. We prove the existence of a selector $\boldsymbol{\eta}^{*}$ which minimizes the functional $J(\boldsymbol{\eta})=\int_{I}\left(F_{\boldsymbol{\eta}}(t)-F^{*}(t)\right)^{2} d t$, where $\boldsymbol{\eta}$ has invariant distribution $F_{\boldsymbol{\eta}}$. Other results pertain to the functional $J_{1}(\boldsymbol{\eta})=\int_{I}\left(P_{\boldsymbol{\eta}} F^{*}(t)-F^{*}(t)\right)^{2} d t$, where $P_{\boldsymbol{\eta}}$ is the Frobenius-Perron operator of $\boldsymbol{\eta}$ acting on distribution functions. We present an algorithm for finding selectors which minimize $J_{1}(\boldsymbol{\eta})$.
\end{abstract}

Department of Mathematics and Statistics, Concordia University, 1455 de Maisonneuve Blvd. West, Montreal, Quebec H3G 1M8, Canada

and

Department of Mathematics, Honghe University, Mengzi, Yunnan 661100, China

E-mails: abraham.boyarsky@concordia.ca, pawel.gora@concordia.ca, zhenyangemail@gmail.com.

\section{INTRODUCTION}

A function $f: X \rightarrow X$ maps every $t \in X$ to only one point $y=f(t)$. There are applications where this is not the case. For example, in economics a consumer's action may not manifest itself in a uniquely determined process. To study such problems we use multivalued functions. A multivalued function $\Gamma$ from a set $X$ to a set $X$, denoted by $\Gamma: X \rightrightarrows X$, is a function from $X$ to the set $2^{X}$ of all subsets of $X$. The theory of such maps is well developed $[2,11]$ and have important applications in rigorous numerics [12], economics [5], dynamical systems [17], and differential relations [1].

The graph of $\Gamma$ is the set: $G=\{(t, s) \in X \times X, s \in \Gamma(t)\}$. Once $\Gamma$ is specified, we consider maps $\boldsymbol{\eta}: X \rightarrow X$ with $\boldsymbol{\eta}(t) \in \Gamma(t)$. Such maps are called selectors. Establishing the existence of continuous selectors in topological spaces has been an area of active interest for more than 60 years $[14,16,1,20,21]$. In the setting of chaotic dynamical systems, however, selectors possessing measure theoretic, rather than topological, properties are of paramount interest. The study of such selectors

Date: May 6, 2015.

2000 Mathematics Subject Classification. 37A05, 37H99, 60J05.

Key words and phrases. multivalued maps, selections of multivalued maps, random maps, absolutely continuous invariant measures.

The research of the authors was supported by NSERC grants. The research of Z. Li is also supported by NNSF of China (No. 11161020 and No. 11361023). 
and their properties was begun in $[7,10]$, where we studied families of selectors that possess absolutely continuous invariant measures (acim). In [7] we studied the properties of the associated attainable probability distribution functions of these families. In simple terms we considered the capacity of a set $G \subset X \times X$ to possess selectors that have acims. This is an entirely new problem and may have application to economics, brain modeling and modeling complex physical processes such as the two-slit experiment of quantum mechanics. In [10] we studied the following problem: given a set $G$ and a selector inside $G$ with invariant distribution $F$, can $F$ be realized by a dynamical system on the boundary of $G$ ? We described a procedure where this can be accomplished for large classes of selectors by using position dependent random maps $([8,19])$ based on the boundaries of the multivalued function. We also studied the extreme points of the attainable distributions of families of selectors that possess acims and showed that in certain settings these extreme points come from selectors that are bang-bang, that is, they take their values in a deterministic way only on the boundaries of the multivalued map.

The inverse Frobenius-Perron operator problem has been solved in a number of ways $[6,9,15]$. None of these methods, however, impose constraints on the dynamical systems (maps) which produce the desired statistical dynamics. The main objective of this note is to solve the inverse Frobenius-Perron problem in special cases under the constraint that the chaotic map which possesses or approximates a targeted density or distribution function has its graph entirely inside a specified region of the space.

Consider, for example, the following economic problem. A desired gross domestic product (GDP) growth for a country is between $2 \%$ and $4 \%$. Let $X=[2,4]$. Let $F^{*}(x)$ be the target distribution function of a sharply peaked probability density function centered at $x=3$. Now we can readily invert the Frobenius-Perron operator for a class of maps without constraints on their images and obtain a map whose dynamics display $F^{*}$. However, in a realistic setting, economists do not want the GDP to range freely across $X$. For example, if the GDP growth is close to $4 \%$, they may want the following year to have lower GDP growth to prevent inflation. On the other hand, if the GDP is $2 \%$ or less, economists would want to stimulate the economy the next year and obtain a GDP of $4 \%$. Such conditions impose constraints on the range of maps that are admissible, forcing the use of boundaries (defined by the graphs of maps) that define a multivalued map and describe the allowable image space for the graphs of selectors.

In many control problems, the boundaries play an important role. For example, in the time optimal problem the control is bang-bang, i.e., entirely on the boundaries of the admissible control region. In the context of selectors, the imposed constraints on the images, may preclude the possibility of solving the inverse Frobenius-Problem problem exactly. For an exact solution the image of the map may be partly or entirely outside the admissible range. In such situations, our target pdf can only be approximated by maps which are entirely or partly on the boundaries of the admissible range defined by the multivalued map. In this note we treat such problems for the first time in the literature.

We consider only the one dimensional situation; that is $X$ is the interval $I=[0,1]$ and $m$ is Lebesgue measure on $I$. We are concerned with the existence of optimal selectors, that is, selectors that minimize a functional. We have a multivalued map $\Gamma: I \rightrightarrows I$. Let $S$ denote a class of selectors. We assume that each map $\boldsymbol{\eta}$ in $S$ 
has an invariant measure $\mu_{\boldsymbol{\eta}}$ with distribution $F_{\boldsymbol{\eta}}\left(F_{\boldsymbol{\eta}}(t)=\mu_{\boldsymbol{\eta}}([0, t])\right.$. Let $F^{*}$ be a target distribution function. We will prove that under certain conditions on $F^{*}$ and $S$, there exists a selector $\boldsymbol{\eta}^{*}$ in $S$ which minimizes the functional:

$$
J(\boldsymbol{\eta})=\int_{I}\left(F_{\boldsymbol{\eta}}(t)-F^{*}(t)\right)^{2} d m(t)
$$

In Section 2 we assume that $\Gamma(t)=\left[\tau_{\ell}(t), \tau_{u}(t)\right]$ is an interval for each $t \in I$ and we define the lower and upper boundary maps to be $\tau_{\ell}(t)$, and $\tau_{u}(t)$, respectively. A simple example is where the admissible class of selectors is characterized by having distributions that are convex combinations of the invariant distributions for the boundary maps, as shown below in Section 2 .

In Section 3 we prove the existence of an optimal selector in a general setting. Although the functional (1) seems simple, it is actually difficult to work with since $F_{\boldsymbol{\eta}}$ is the solution of a functional equation (Frobenius-Perron equation) involving the unknown selector $\boldsymbol{\eta}$. In Sections 4 and 5 we consider the simpler functional

$$
J_{1}(\boldsymbol{\eta})=\int_{I}\left(P_{\boldsymbol{\eta}} F^{*}(t)-F^{*}(t)\right)^{2} d m(t),
$$

where $P_{\boldsymbol{\eta}}$ is the Frobenius-Perron operator of $\boldsymbol{\eta}$ acting on distribution functions [4] and present a complete solution to the optimal problem for symmetric maps. Since we can write

$$
F_{\boldsymbol{\eta}}-F^{*}=\left(F_{\boldsymbol{\eta}}-P_{\boldsymbol{\eta}} F_{\boldsymbol{\eta}}\right)+\left(P_{\boldsymbol{\eta}} F_{\boldsymbol{\eta}}-P_{\boldsymbol{\eta}} F^{*}\right)+\left(P_{\boldsymbol{\eta}} F^{*}-F^{*}\right),
$$

$J_{1}$ is a part of $J$. $J_{1}$ also has the property that if the minimum of $J$ is 0 for the selector $\boldsymbol{\eta}^{*}$, then the minimum of $J_{1}$ is also 0 for $\boldsymbol{\eta}^{*}$.

In Section 5 we define a class of target distribution functions and a class of admissible selectors for which we can apply a practical algorithm which achieves an optimal selector. An example will show that the optimal selectors can have their images on the boundaries of the admissible region.

\section{SIMPLE EXAMPLE}

Let $\mathcal{P}$ be a partition of $I$ into a finite number of intervals. Let the lower and upper boundary maps $\tau_{\ell}, \tau_{u}: I \rightarrow I$ be two piecewise expanding maps on $\mathcal{P}$, i.e., piecewise monotonic, piecewise $C^{2}$ maps with $\inf \left|\tau^{\prime}\right|>1$. Then, the graph of $\Gamma$ is the region $G \subset I \times I$ bounded by the graphs of $\tau_{\ell}$ and $\tau_{u}$. Any map $\boldsymbol{\eta}: I \rightarrow I$ whose graph is in $G$ is a selector. Since $\tau_{\ell}, \tau_{u}$ are piecewise expanding they possess acims ([3]) $\mu_{\ell}$ and $\mu_{u}$, respectively. Let $F_{\ell}$ and $F_{u}$ denote the probability distribution functions of $\mu_{\ell}$ and $\mu_{u}$, respectively. From [7] we know that, for any convex combination $F$ of $F_{\ell}$ and $F_{u}$, we can find a selector $\boldsymbol{\eta}$ such that $F$ is the $\boldsymbol{\eta}$-invariant distribution function. Let $F_{\boldsymbol{\eta}}=\lambda F_{\ell}+(1-\lambda) F_{u}$. Then,

$$
\begin{aligned}
J(\boldsymbol{\eta}) & =\int_{I}\left(\lambda F_{\ell}+(1-\lambda) F_{u}-F^{*}\right)^{2} d m \\
& =\lambda^{2} \int_{I}\left(F_{\ell}-F_{u}\right)^{2} d m+2 \lambda \int_{I}\left(F_{\ell}-F_{u}\right)\left(F_{u}-F^{*}\right) d m+\int_{I}\left(F_{u}-F^{*}\right)^{2} d m
\end{aligned}
$$

To minimize, we differentiate in $\lambda$ and obtain the optimal $\lambda^{*}$ :

$$
\lambda^{*}=\frac{\int_{I}\left(F_{u}-F_{\ell}\right)\left(F_{u}-F^{*}\right) d m}{\int_{I}\left(F_{\ell}-F_{u}\right)^{2} d m} .
$$


If $\lambda^{*}$ does not satisfy $0 \leq \lambda^{*} \leq 1$, we choose $\lambda^{*}$ equal to 0 or 1 . Note that, by the Cauchy-Schwarz inequality, $\lambda^{*} \leq 1$ if

$$
\int_{I}\left(F_{u}-F^{*}\right)^{2} d m \leq \int_{I}\left(F_{u}-F_{\ell}\right)^{2} d m .
$$

\section{EXISTENCE THEOREM}

In this section, we consider the case where the boundary maps $\tau_{\ell}$ and $\tau_{u}$ are piecewise expanding and piecewise onto on the partition $\mathcal{P}=\left\{I_{1}, I_{2}\right\}$ of $I$, where $I_{1}=[0,1 / 2]$ and $I_{2}=[1 / 2,1]$. We assume that both boundary maps are increasing on $I_{1}$ and decreasing on $I_{2}$.

Let $\mathcal{T}$ be the class of maps $\boldsymbol{\eta}: I \rightarrow I$ satisfying the following conditions:

(a) Each $\boldsymbol{\eta} \in \mathcal{T}$ is piecewise expanding on $\mathcal{P}$ with the same monotonicity as the boundary maps on elements of $\mathcal{P}$.

(b) There is a $\alpha>1$ such that $\left|\boldsymbol{\eta}^{\prime}\right|>\alpha$ whenever $\left|\boldsymbol{\eta}^{\prime}\right|$ exists, for all $\boldsymbol{\eta} \in \mathcal{T}$.

(c) There is a $M>0$ such that $V_{[0,1]} \boldsymbol{\eta}^{\prime} \leq M$, for all $\boldsymbol{\eta} \in \mathcal{T}$, where $V_{[0,1]} f$ denotes the total variation of the function $f$ on $[0,1]$.

(d) $\tau_{\ell}(t) \leq \boldsymbol{\eta}(t) \leq \tau_{u}(t)$ for all $\boldsymbol{\eta} \in \mathcal{T}, t \in[0,1]$.

Then, any $\boldsymbol{\eta} \in \mathcal{T}$ preserves a distribution function $F$ whose density function is supported on all of $I$, i.e., $F^{\prime}>0$ a.e., [3]. From [4] we know that $F$ satisfies the equation $F(t)=F\left(\boldsymbol{\eta}_{1}^{-1}(t)\right)+1-F\left(\boldsymbol{\eta}_{2}^{-1}(t)\right), t$ a.e., where $\boldsymbol{\eta}_{i}=\left.\boldsymbol{\eta}\right|_{I_{i}}, i=1,2$.

We define the set of attainable distribution functions, $\mathbf{D}=\{F \mid F$ is a distribution function preserved by some selector $\boldsymbol{\eta} \in \mathcal{T}\}$. For a given target distribution function $F^{*}$, we will show the existence of a selector minimizing the functional $J$ defined in (1)

$$
J(\boldsymbol{\eta})=\int_{I}\left(F_{\boldsymbol{\eta}}-F^{*}\right)^{2} d m
$$

We first prove the existence of an optimal distribution function in the set $\mathbf{D}$.

Lemma 1. The set $\mathbf{D}$ is compact in $L^{1}$ and convex.

Proof. For each $F \in \mathbf{D}$, we have both $|F| \leq 1$ and $V_{[0,1]} F=1$. Thus, by Helly's Theorem the set $\mathbf{D}$ is pre-compact in $L^{1}$. We show that $\mathbf{D}$ is closed. Let $F_{n} \rightarrow F$, $n=1,2, \ldots$, be a convergent sequence of distributions in $\mathbf{D}$. Let $\left\{\boldsymbol{\eta}_{n}\right\} \subset \mathcal{T}$ be the sequence of corresponding selectors. Let us consider the sequence of their derivatives $\left\{\boldsymbol{\eta}_{n}^{\prime}\right\}$. It follows from the assumption (c) of $\mathcal{T}$ and Helly's theorem that $\left\{\boldsymbol{\eta}_{n}^{\prime}\right\}$ contains a subsequence convergent to a function $\boldsymbol{\eta}^{\prime}$, which satisfies assumptions b), c), d). Its integral is the map $\boldsymbol{\eta}$ which is clearly in $\mathcal{T}$. Because of acim stability of $\boldsymbol{\eta}$ ( [3]) its invariant distribution is $F$, the limit of the $F_{n}$ 's.

Now we will prove that $\mathbf{D}$ is convex. Let $F^{(1)}, F^{(2)} \in \mathbf{D}$ be two different distributions which are preserved by selectors $\gamma^{(1)}$ and $\gamma^{(2)}$, respectively. Let $0<$ $\lambda<1$ and $F=\lambda F^{(1)}+(1-\lambda) F^{(2)}$.

We define the inverse of each branch of a new selector $\boldsymbol{\eta}$ by

$$
\boldsymbol{\eta}_{i}^{-1}(t)=F^{-1}\left(\lambda F^{(1)}\left(\left(\gamma_{i}^{(1)}\right)^{-1}(t)\right)+(1-\lambda) F^{(2)}\left(\left(\gamma_{i}^{(2)}\right)^{-1}(t)\right)\right),
$$

where $\boldsymbol{\eta}_{i}^{-1}=\left.\boldsymbol{\eta}\right|_{I_{i}}{ }^{-1},\left(\boldsymbol{\gamma}_{i}^{(1)}\right)^{-1}=\left(\left.\boldsymbol{\gamma}^{(1)}\right|_{I_{i}}\right)^{-1},\left(\boldsymbol{\gamma}_{i}^{(2)}\right)^{-1}=\left(\left.\boldsymbol{\gamma}^{(2)}\right|_{I_{i}}\right)^{-1}, i=1,2$. The selector $\boldsymbol{\eta}$ defined in this way has the same monotonicity and number of branches as $\boldsymbol{\gamma}^{(1)}$ and $\boldsymbol{\gamma}^{(2)}$. Invoking the proof of Theorem 1 of [7], we can show that $\boldsymbol{\eta}$ preserves $F$. 
To show that $F \in \mathbf{D}$ it suffices to prove that $\boldsymbol{\eta}$ is a selector, that is, that its graph is between the graphs of selectors $\gamma^{(1)}$ and $\gamma^{(2)}$. There are two possibilities.

(I): If $\gamma_{i}^{(1)} \leq \gamma_{i}^{(2)}, i=1,2$, then we can think of $\gamma^{(1)}$ and $\gamma^{(2)}$ as boundary maps, and the lemma can be proved as in Theorem 1 of [7].

(II): (I) is not the case, which means one of the selectors $\gamma^{(1)}$ and $\gamma^{(2)}$ is not always above the other. Let us consider the first branch. The proof for the second branch is similar. Let us further assume that on an interval $[a, b] \subseteq I$, we have $\left(\gamma_{1}^{(1)}\right)^{-1} \geq\left(\gamma_{1}^{(2)}\right)^{-1}$ (the proof for the case $\left(\gamma_{1}^{(1)}\right)^{-1} \leq\left(\gamma_{1}^{(2)}\right)^{-1}$ is similar), and $\left(\gamma_{1}^{(1)}\right)^{-1}(a)=\left(\gamma_{1}^{(2)}\right)^{-1}(a),\left(\gamma_{1}^{(1)}\right)^{-1}(b)=\left(\gamma_{1}^{(2)}\right)^{-1}(b)$. Now, for $t \in[a, b]$, it follows from equation (4) that

$$
F\left(\boldsymbol{\eta}_{1}^{-1}(t)\right)=\lambda F^{(1)}\left(\left(\gamma_{1}^{(1)}\right)^{-1}(t)\right)+(1-\lambda) F^{(2)}\left(\left(\gamma_{1}^{(2)}\right)^{-1}(t)\right) .
$$

Since $F^{(1)}$ and $F^{(2)}$ are non-decreasing, we have

$$
\begin{aligned}
& \lambda F^{(1)}\left(\left(\gamma_{1}^{(2)}\right)^{-1}(t)\right)+(1-\lambda) F^{(2)}\left(\left(\gamma_{1}^{(2)}\right)^{-1}(t)\right) \\
& \leq F\left(\boldsymbol{\eta}_{1}^{-1}(t)\right) \leq \lambda F^{(1)}\left(\left(\gamma_{1}^{(1)}\right)^{-1}(t)\right)+(1-\lambda) F^{(2)}\left(\left(\gamma_{1}^{(1)}\right)^{-1}(t)\right),
\end{aligned}
$$

which is equivalent to

$$
F\left(\left(\gamma_{1}^{(2)}\right)^{-1}(t)\right) \leq F\left(\boldsymbol{\eta}_{1}^{-1}(t)\right) \leq F\left(\left(\gamma_{1}^{(1)}\right)^{-1}(t)\right)
$$

implying that $\boldsymbol{\eta}_{1}^{-1}$ defined in (4) has its graph between those of $\left(\boldsymbol{\gamma}_{1}^{(1)}\right)^{-1}$ and $\left(\gamma_{1}^{(2)}\right)^{-1}$. Thus, $\boldsymbol{\eta}$ defined by equation (4) is located between $\boldsymbol{\gamma}^{(1)}$ and $\boldsymbol{\gamma}^{(2)}$. Since $\gamma^{(1)}$ and $\gamma^{(1)}$ are selectors, $\boldsymbol{\eta}$ is also a selector. This shows that $F \in \mathbf{D}$ and completes the proof of the lemma.

Remark 2. The above proof can be generalized to maps and selectors having more than two branches and not necessarily piecewise onto as well as to random maps $[8,19]$.

Let $d(\cdot, \cdot)$ be the metric induced by the norm on $L^{2}$. We now present the main result of this section.

Theorem 3. There exists a unique point $\bar{F}$ in $\mathbf{D}$ such that

$$
d\left(F^{*}, \bar{F}\right)=d\left(F^{*}, \mathbf{D}\right)
$$

and there exists a selector $\boldsymbol{\eta} \in \mathcal{T}$ which preserves the distribution $\bar{F}$ and minimizes the functional $J$.

Proof. Since D consists of uniformly bounded functions it is compact and convex in $L^{2}$. The existence of a unique $\bar{F}$ follows by strict convexity of $L^{2}$. The existence of a corresponding $\boldsymbol{\eta}$ follows from the definition of $\mathbf{D}$, although there might be more than one selector preserving the distribution $\bar{F}$.

\section{SYMMETRIC OPTIMAL SELECTORS}

In this section we consider selectors that are symmetric and prove the existence of an optimal selector. Let the boundary maps $\tau_{s}:[0,1] \rightarrow[0,1], s \in\{\ell, u\}$, satisfy

(a) $\tau_{s}$ is continuous and monotonic on $I_{1}=[0,1 / 2]$ and on $I_{2}=[1 / 2,1]$ with $\tau_{s}(0)=\tau_{s}(1)=0$ and $\tau_{s}(1 / 2)=1, s \in\{\ell, u\}$. Also, $\tau_{\ell} \leq \tau_{u}$.

(b) $\tau_{s}(t)=\tau_{s}(1-t)$ for all $t \in[0,1]$, i.e., $\tau_{s}$ 's are symmetric with respect $t=1 / 2$, $s \in\{\ell, u\}$. 
Let $F^{*}$ be a distribution with $f^{*}=\left(F^{*}\right)^{\prime}>0$ on all of $[0,1]$. We are looking for a map $\tau_{\ell} \leq \boldsymbol{\eta} \leq \tau_{u}$ satisfying conditions (a) and (b) and which minimizes the functional $J_{1}(\overline{\boldsymbol{\eta}})=\int_{I}\left(P_{\boldsymbol{\eta}}\left(F^{*}\right)-F^{*}\right)^{2} d m$ defined in $(2)$. If we define $x(t)=\boldsymbol{\eta}_{1}^{-1}(t)$ : $[0,1] \rightarrow[0,1 / 2]\left(\right.$ where $\left.\boldsymbol{\eta}_{1}=\boldsymbol{\eta}_{\mid I_{1}}\right)$, then we have

$$
J_{1}(\boldsymbol{\eta})=\int_{I}\left[F^{*}(x(t))+1-F^{*}(1-x(t))-F^{*}(t)\right]^{2} d m(t) .
$$

It is enough to find the $x^{*}$ that minimizes $J_{1}(\boldsymbol{\eta})$. Since $\boldsymbol{\eta}(t)$ is symmetric, $x(t)=$ $\boldsymbol{\eta}_{1}^{-1}(t)$ determines $\boldsymbol{\eta}(t)$ uniquely on $[0,1]$. Hence we can write $J_{1}(\boldsymbol{\eta})=J_{1}(x)$.

We have the following obvious proposition:

Proposition 4. If for each $t \in[0,1], x^{*}(t)$ is admissible and such that $\left[F^{*}\left(x^{*}(t)\right)+\right.$ $\left.1-F^{*}\left(1-x^{*}(t)\right)-F^{*}(t)\right]^{2}$ is minimal, then $J_{1}\left(x^{*}\right)$ is the minimum.

We now prove:

Proposition 5. The optimal admissible solution $x^{*}(t)=:[0,1] \rightarrow[0,1 / 2]$ is an increasing continuous union of pieces which satisfy the Frobenius-Perron operator equation for distributions [4]:

$$
\left[F^{*}(x)+1-F^{*}(1-x)-F^{*}(t)\right]^{2}=0,
$$

together with pieces of the graphs of $\tau_{\ell}^{-1}$ and $\tau_{u}^{-1}$.

Proof. First, we will show that equation (5) has a unique solution. We rewrite equation (5) as

$$
F^{*}(1-x)-F^{*}(x)=1-F^{*}(t) .
$$

Since $F^{* \prime}>0$, both sides are strictly decreasing functions. The left hand side equals 1 for $x=0$ and equals 0 for $x=1 / 2$. The right hand side equals 1 for $t=0$ and 0 for $t=1$. This means that for any $t \in[0,1]$ there is exactly one $x \in[0,1 / 2]$ satisfying equation (6). Thus, $x(t)$ is a continuous strictly increasing function from $[0,1]$ onto $[0,1 / 2]$.

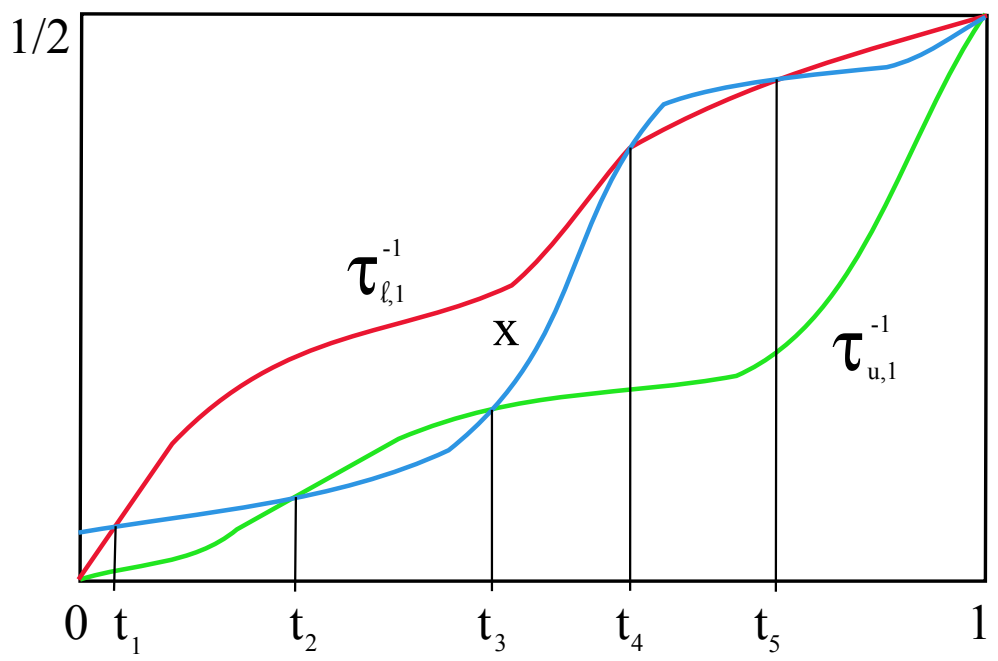

FiguRE 1. Lower and upper inverses and the solution $x(t)$ of (5) 
The remainder of the proof is motivated by Figure 1 which shows the first branches of the inverses of the lower boundary map $\tau_{\ell, 1}^{-1}$ (red) and of upper boundary map $\tau_{u, 1}^{-1}$ (green) and the curve $\mathrm{x}(\mathrm{t})$, the unique solution of $G(t, x)=\left[F^{*}(x)+\right.$ $\left.1-F^{*}(1-x)-F^{*}(t)\right]^{2}=0$ (blue). Note that $\tau_{\ell} \leq \tau_{u}$ implies $\tau_{\ell, 1}^{-1} \geq \tau_{u, 1}^{-1}$. Since $G(t, x)$ is non-negative and the value of $G(t, x)$ at $x(t)$ is $0, x(t)$ is the minimum point of $G(t, x)$. We have

$$
\frac{\partial G(t, x)}{\partial x}=2\left[F^{*}(x)+1-F^{*}(1-x)-F^{*}(t)\right]\left(f^{*}(x)+f^{*}(1-x)\right) .
$$

Since $f^{*}>0, x(t)$ is the only zero of $\frac{\partial G(t, x)}{\partial x}$ for any fixed $t$. Thus, for each fixed $t$ the graph of $G(t, x)$ is similar to that of the parabola $x^{2}$.

Thus, the surface $\left[F^{*}(x)+1-F^{*}(1-x)-F^{*}(t)\right]^{2}$ looks like a river valley with the graph of $x(t)$ (blue) the river at the bottom. Now, we construct the optimal admissible solution $x^{*}(t)$. We set

$$
x^{*}(t)=\max \left\{\min \left\{x(t), \tau_{\ell, 1}^{-1}(t)\right\}, \tau_{u, 1}^{-1}(t)\right\},
$$

i.e.,

$$
x^{*}(t)= \begin{cases}x(t) & \text { if } \tau_{u, 1}^{-1}(t) \leq x(t) \leq \tau_{\ell, 1}^{-1}(t) ; \\ \tau_{\ell, 1}^{-1}(t) & \text { if } x(t) \geq \tau_{\ell, 1}^{-1}(t) \\ \tau_{u, 1}^{-1}(t) & \text { if } x(t) \leq \tau_{u, 1}^{-1}(t) .\end{cases}
$$

Whenever $x^{*}(t) \neq x(t)$, the graph of $x^{*}(t)$ separates the curve $x(t)$ from all other admissible points so $x^{*}(t)$ has the lowest admissible values. $x^{*}(t)$ constructed in this way is continuous, increasing and for each point $t$ has the lowest admissible value. Thus, $x^{*}(t)$ minimizes $J_{1}(x)$ by Proposition 4 .

In the example shown in Figure 1 the optimal solution $x^{*}(t)$ is defined as follows:

$$
x^{*}(t)= \begin{cases}\tau_{\ell, 1}^{-1}(t), & \text { for } t \in\left[0, t_{1}\right] ; \quad \text { (red) } \\ x(t), & \text { for } t \in\left[t_{1}, t_{2}\right] ; \quad \text { (blue) } \\ \tau_{u, 1}^{-1}(t), & \text { for } t \in\left[t_{2}, t_{3}\right] ; \quad \text { (green) } \\ x(t), & \text { for } t \in\left[t_{3}, t_{4}\right] ; \text { (blue) } \\ \tau_{\ell, 1}^{-1}(t), & \text { for } t \in\left[t_{4}, t_{5}\right] ; \text { (red) } \\ x(t), & \text { for } t \in\left[t_{5}, 1\right] .\end{cases}
$$

It is interesting to summarize that segments of the optimal solution are on the boundaries of the admissible region.

We will now consider slightly more general cases. The first case has boundary maps which are tent-shaped and the other with the boundary maps shaped like the $2 x(\bmod 1) \operatorname{map}$.

A: Tent-shaped maps: Let $\tau_{s}:[0,1] \rightarrow[0,1], s \in\{\ell, u\}$, satisfy

(a) $\tau_{s}$ is continuous and monotonic on $[0,1 / 2]$ and on $[1 / 2,1]$ with $\tau_{s}(0)=\tau_{s}(1)=$ 0 and $\tau_{s}(1 / 2)=1, s \in\{\ell, u\}$. Also, $\tau_{\ell} \leq \tau_{u}$.

(b) There exists a strictly decreasing continuous function $w:[0,1 / 2] \rightarrow[1 / 2,1]$, $w(0)=1, w(1 / 2)=1 / 2$ such that $\tau_{s, 1}(t)=\tau_{s, 2}(w(t))$ for all $t \in[0,1 / 2], s \in\{\ell, u\}$.

Let $F^{*}$ be a target distribution with $f^{*}=F^{* \prime}>0$ on all of $[0,1]$. We are looking for a map $\tau_{\ell} \leq \boldsymbol{\eta} \leq \tau_{u}$ satisfying conditions (a) and (b) and which minimizes the 


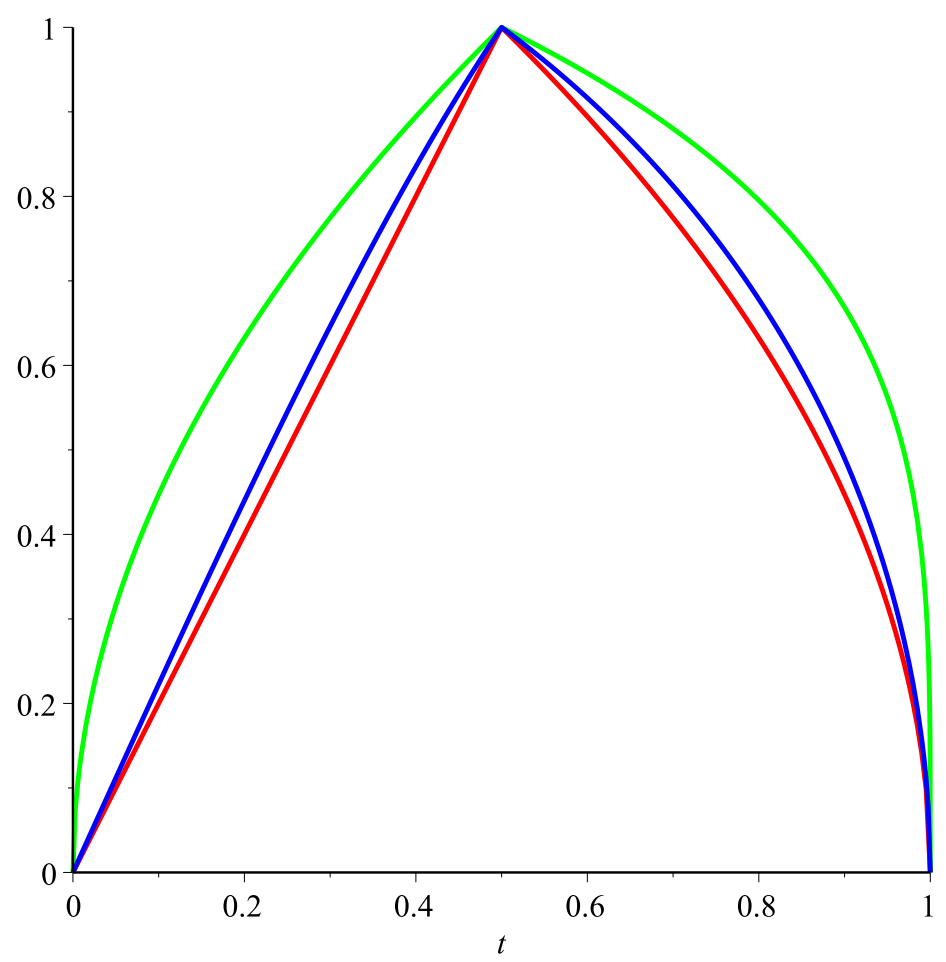

Figure 2. Maps $\tau_{\ell}$ (red), $\tau_{u}$ (green) and the optimal solution $\boldsymbol{\eta}$ (blue) for $F^{*}(t)=t^{2}$.

functional $J_{1}(\boldsymbol{\eta})$. If we define $x(t)=\boldsymbol{\eta}_{1}^{-1}:[0,1] \rightarrow[0,1 / 2]$, then we have

$$
J_{1}(\boldsymbol{\eta})=\int_{I}\left[F^{*}(x(t))+1-F^{*}(w(x(t)))-F^{*}(t)\right]^{2} d m(t) .
$$

Since $\boldsymbol{\eta}(t)$ satisfies condition (b), $x(t)=\boldsymbol{\eta}_{1}^{-1}(t)$ determines $\boldsymbol{\eta}(t)$ uniquely on $[0,1]$. Hence, we can write $J_{1}(\boldsymbol{\eta})=J_{1}(x)$. It is enough to find $x^{*}$ that minimizes $J_{1}(x)$.

Proposition 6. The optimal admissible solution $x^{*}(t)=:[0,1] \rightarrow[0,1 / 2]$ is an increasing continuous union of pieces which satisfy the Frobenius-Perron operator equation for distributions [4]:

$$
\left[F^{*}(x)+1-F^{*}(w(x))-F^{*}(t)\right]^{2}=0
$$

together with pieces of the graphs of $\tau_{\ell}^{-1}$ and $\tau_{u}^{-1}$.

The proof is almost identical to that of Proposition 5. 


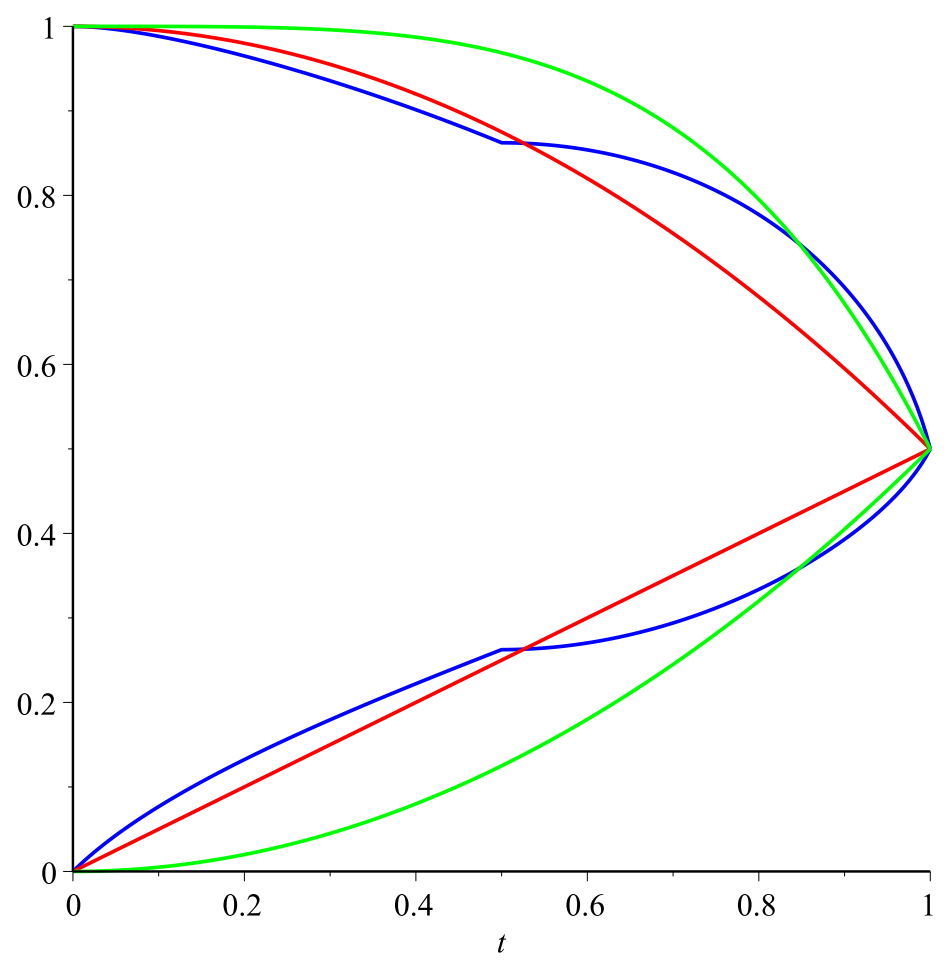

FiguRE 3. Inverse branches of $\tau_{\ell}$ (red), $\tau_{u}$ (green) and of solution $x$ (blue) for $F^{*}(t)$ defined by equation ( 8 ).

\section{Example 7.}

Let $w(t)=1-2 t^{2}, t \in[0,1 / 2]$. Let $\tau_{\ell, 1}(t)=2 t, \tau_{u, 1}(t)=\sqrt{2 t}$ for $0 \leq t \leq 1 / 2$ and define

$$
\tau_{s}(t)= \begin{cases}\tau_{s, 1}(t) & , \text { for } 0 \leq t \leq 1 / 2 \\ \tau_{s, 1}\left(w^{-1}(t)\right) & , \text { for } 1 / 2<t \leq 1\end{cases}
$$

$s \in\{\ell, u\}$, see the red and green graphs in Figure 2. For the target distribution $F^{*}(t)=t^{2}$ we can solve the equation (7) and obtain $x(t)=\frac{1}{4} \sqrt{10-2 \sqrt{25-16 t^{2}}}$. Then, $\boldsymbol{\eta}_{1}(t)=x^{-1}(t)=t \sqrt{5-4 t^{2}}$ and $\boldsymbol{\eta}_{2}(t)=\boldsymbol{\eta}_{1}\left(w^{-1}(t)\right)$. See the blue graph in Figure 2. In this case the map $\boldsymbol{\eta}$ is between maps $\tau_{\ell}$ and $\tau_{u}$ and it is the selector which minimizes the functional $J_{1}(\boldsymbol{\eta})$. It minimizes the functional $J(\boldsymbol{\eta})$ as well.

For the target distribution

$$
F^{*}(t)= \begin{cases}t & , \text { for } 0 \leq t \leq 1 / 2 \\ 2 t^{2}-2 t+1 & , \text { for } 1 / 2<t \leq 1\end{cases}
$$

we found $x(t)$, the solution of equation (7), numerically. The graphs presented in Figure 3 are: $\tau_{\ell, 1}^{-1}$ and $\tau_{\ell, 2}^{-1}$ in red, $\tau_{u, 1}^{-1}$ and $\tau_{u, 2}^{-1}$ in green, $x$ and $w \circ x$ in blue. This time the graph of $x$ is not entirely between the graphs of $\tau_{\ell, 1}^{-1}$ and $\tau_{u, 1}^{-1}$. The optimal 
solution is

$$
x^{*}(t)= \begin{cases}\tau_{\ell, 1}^{-1}(t) & , \text { for } 0 \leq t \leq 0.5259574806 \\ x(t) & , \text { for } 0.5259574806<t \leq 0.846817465580226 \\ \tau_{u, 1}^{-1}(t) & , \text { for } 0.846817465580226<t \leq 1\end{cases}
$$

and the optimal selector is

$$
\boldsymbol{\eta}^{*}(t)= \begin{cases}\left(x^{*}\right)^{-1}(t) & , \text { for } 0 \leq t \leq 1 / 2 \\ \left(x^{*}\right)^{-1}\left(w^{-1}(t)\right) & , \text { for } 1 / 2<t \leq 1\end{cases}
$$

B: $2 x(\bmod 1)$-shaped maps: Let $\tau_{s}:[0,1] \rightarrow[0,1], s \in\{\ell, u\}$, satisfy:

(a) $\tau_{s}$ is continuous and monotonic on $[0,1 / 2]$ and on $[1 / 2,1]$ with $\tau_{s}(0)=$ $\tau_{s}\left(1 / 2^{+}\right)=0$ and $\tau_{s}\left(1 / 2^{-}\right)=\tau_{s}(1)=1, s \in\{\ell, u\}$. Also $\tau_{\ell} \leq \tau_{u}$.

(b) There exists a strictly increasing continuous function $w:[0,1 / 2] \rightarrow[1 / 2,1]$, $w(0)=1 / 2, w(1 / 2)=1$ such that $\tau_{s, 1}(t)=\tau_{s, 2}(w(t))$ for all $t \in[0,1 / 2], s \in\{\ell, u\}$.

Let $F^{*}$ be a distribution with $f^{*}=\left(F^{*}\right)^{\prime}>0$ on all of $[0,1]$. We are looking for a map $\tau_{\ell} \leq \boldsymbol{\eta} \leq \tau_{u}$ satisfying conditions (a) and (b) and which minimizes the functional $J_{1}(\boldsymbol{\eta})=\int_{I}\left(P_{\boldsymbol{\eta}}\left(F^{*}\right)-F^{*}\right)^{2} d m$. If we define $x(t)=\boldsymbol{\eta}_{1}^{-1}(t):[0,1] \rightarrow$ $[0,1 / 2]$, then we have

$$
J_{1}(\boldsymbol{\eta})=\int_{I}\left[F^{*}(x(t))+F^{*}(w(x(t)))-F^{*}(1 / 2)-F^{*}(t)\right]^{2} d m(t) .
$$

The form of $P_{\boldsymbol{\eta}}\left(F^{*}\right)$ is different than before since the shape of the maps we now consider is different. Since $\boldsymbol{\eta}(t)$ satisfies condition $(\mathrm{b}), x(t)=\boldsymbol{\eta}_{1}^{-1}(t)$ determines $\boldsymbol{\eta}(t)$ uniquely on $[0,1]$. Hence, we can write $J_{1}(\boldsymbol{\eta})=J_{1}(x)$. It is enough to find $x^{*}$ that minimizes $J_{1}(x)$.

Proposition 8. The optimal admissible solution $x^{*}(t)=:[0,1] \rightarrow[0,1 / 2]$ is an increasing continuous union of pieces which satisfy the Frobenius-Perron operator equation for distributions:

$$
\left[F^{*}(x)+F^{*}(w(x))-F^{*}(1 / 2)-F^{*}(t)\right]^{2}=0
$$

together with pieces of the graphs of $\tau_{\ell}^{-1}$ and $\tau_{u}^{-1}$.

Again, the proof follows closely that of Proposition 5.

\section{An ALGORITHM}

We consider maps $\tau_{\ell}, \tau_{u}\left(\tau_{\ell} \leq \tau_{u}\right)$ and $\boldsymbol{\eta}$ shaped like $2 x(\bmod 1)$, i.e., if $\rho$ denotes any of these maps we have: both branches $\rho_{1}, \rho_{2}$ are increasing, $\rho_{1}(0)=\rho_{2}(1 / 2)=$ $0, \rho_{1}(1 / 2)=\rho_{2}(1)=1$ and $\rho_{1}(t) \geq t, 0 \leq t \leq 1 / 2, \rho_{2}(t) \leq t, 1 / 2 \leq t \leq 1$. See Figure 4. We also assume $\rho_{1}^{\prime}>1, \rho_{2}^{\prime}>1$. We let $x(t)=\boldsymbol{\eta}_{1}^{-1}(t)$ and $y(t)=\boldsymbol{\eta}_{2}^{-1}(t)$, $0 \leq t \leq 1$. Thus, $x^{\prime}<1$ and $y^{\prime}<1$.

The fixed point Frobenius-Perron operator equation we want satisfied as closely as possible is

$$
F^{*}(t)=F^{*}(x(t))+F^{*}(y(t))-F^{*}(1 / 2),
$$

where $F^{*}$ is a given target distribution function. We assume $f^{*}=\left(F^{*}\right)^{\prime}>0$ on all of $[0,1]$.

We present an algorithm which, for any given $y(t)$, produces a sequence of selectors $\boldsymbol{\eta}^{(n)}$ with diminishing values of $J_{1}\left(\boldsymbol{\eta}^{(n)}\right)$.

We assume: 

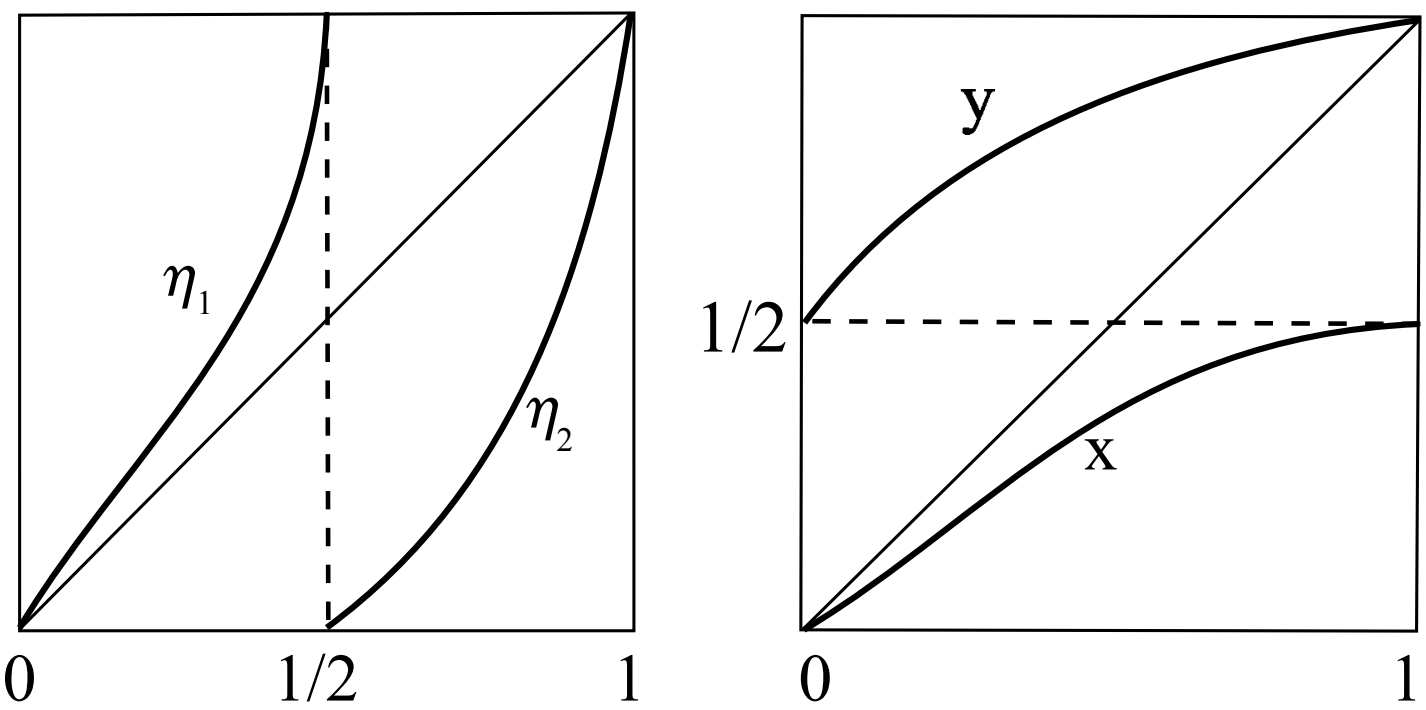

FigURE 4. $\boldsymbol{\eta}_{1}, \boldsymbol{\eta}_{2}$ and their inverses.

(i) $\left(F^{*}\right)^{\prime}$ is decreasing;

(ii) the inverses $\tau_{\ell, 1}^{-1}$ and $\tau_{u, 1}^{-1}$ of the boundary maps satisfy the following condition related to equation (10): the functions

$$
y_{s}(t)=F^{*-1}\left[F^{*}(t)-F^{*}\left(\tau_{s, 1}^{-1}(t)\right)+F^{*}(1 / 2)\right], s \in\{\ell, u\}
$$

satisfy

$$
0 \leq y_{s}^{\prime}(t) \leq 1, t \in[0,1], s \in\{\ell, u\}
$$

The algorithm, with some modifications, can be applied even if condition (ii) does not hold. The first loop of the procedure is illustrated in Figures 5, 6 and 7.

Let $\boldsymbol{\eta}^{(0)}$ be any selector and let $y^{(1)}(t)=\left(\boldsymbol{\eta}_{2}^{(0)}\right)^{-1}(t)$. Then, $y^{(1)}$ satisfies the assumptions of Proposition 9, which is proved in the sequel. Using equation (10), we define the corresponding temporary inverse

$$
x^{(2, T)}(t)=\left(F^{*}\right)^{-1}\left[F^{*}(t)-F^{*}\left(y^{(1)}(t)\right)+F^{*}(1 / 2)\right] .
$$

In Figure 5 we show the initial inverse $y^{(1)}(t)$ and temporary $x^{(2, T)}(t)$. On intervals $\left[0, t_{1}\right]$ and $\left[t_{2}, t_{3}\right]$ this pair is already optimal, i.e., they contribute 0 to the value of the functional $J_{1}\left(\boldsymbol{\eta}^{(1, T)}\right)$ where

$$
\boldsymbol{\eta}^{(1, T)}(\theta)= \begin{cases}\left(x^{(2, T)}\right)^{-1}(\theta), & 0 \leq \theta \leq 1 / 2 \\ \left(y^{(1)}\right)^{-1}(\theta), & 1 / 2<\theta \leq 1\end{cases}
$$

On intervals $\left[t_{1}, t_{2}\right]$ and $\left[t_{3}, 1\right]$ the temporary $x^{(2, T)}(t)$ is outside the region defined by the boundary maps and is, therefore, inadmissible. We correct it as follows: let

$$
x^{(2)}(t)=\max \left\{\min \left\{x^{(2, T)}(t), \tau_{\ell, 1}^{-1}(t)\right\}, \tau_{u, 1}^{-1}(t)\right\} .
$$




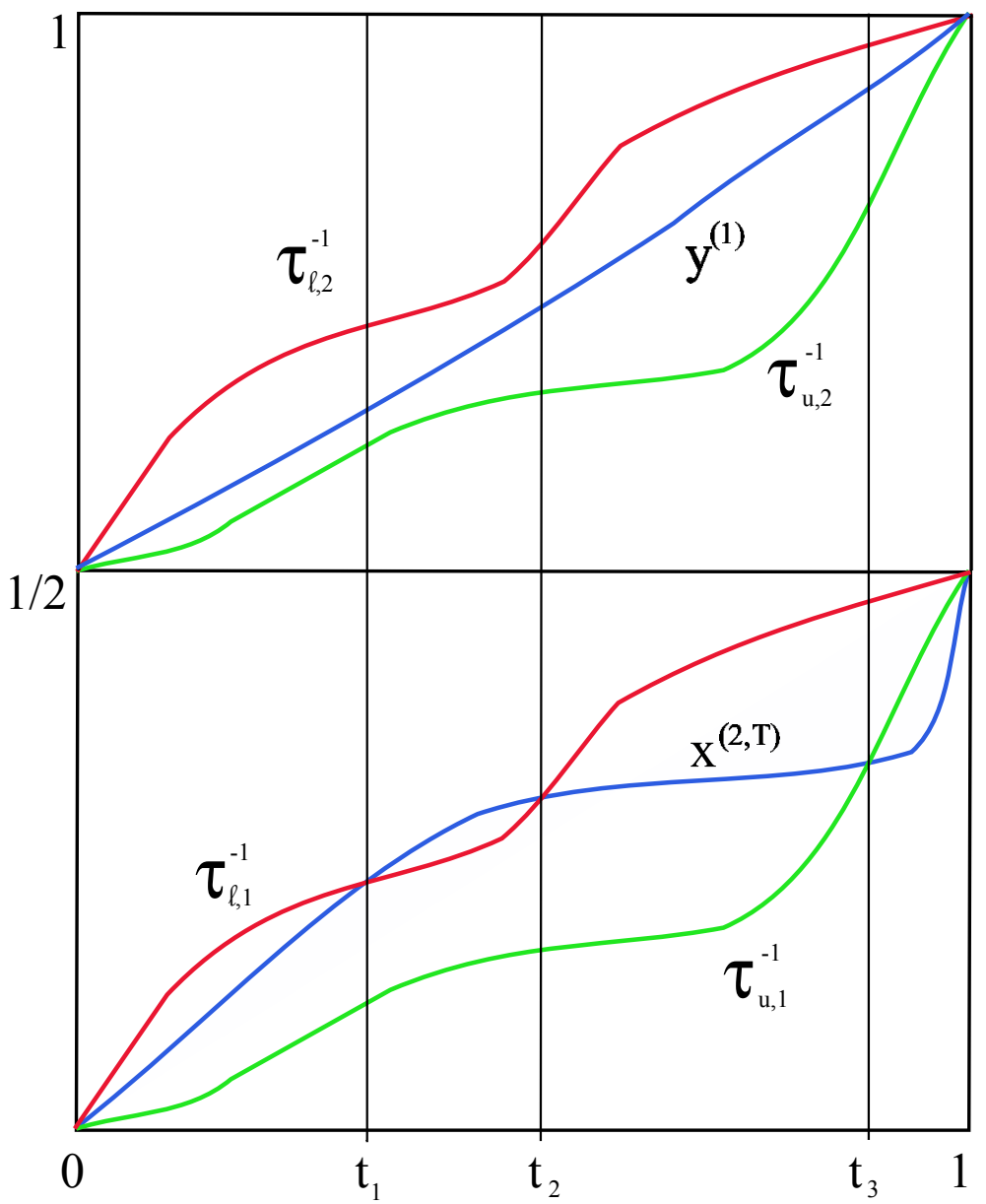

Figure 5. The initial inverse $y^{(1)}(t)$ and the corresponding temporary inverse $x^{(2, T)}(t)$.

It can be proven the same way as in Proposition 5 that, for given $y^{(1)}$, this choice of $x^{(2)}$ produces the minimal possible value of $J_{1}\left(\boldsymbol{\eta}^{(1)}\right)$, where

$$
\boldsymbol{\eta}^{(1)}(\theta)= \begin{cases}\left(x^{(2)}\right)^{-1}(\theta), & 0 \leq \theta \leq 1 / 2 \\ \left(y^{(1)}\right)^{-1}(\theta), & 1 / 2<\theta \leq 1\end{cases}
$$

In particular, $J_{1}\left(\boldsymbol{\eta}^{(1)}\right) \leq J_{1}\left(\boldsymbol{\eta}^{(0)}\right)$. The map $x^{(2)}$ is shown in Figure 6 . Now we produce the temporary inverse corresponding to $x^{(2)}(t)$ using equation (10):

$$
y^{(3, T)}(t)=\left(F^{*}\right)^{-1}\left[F^{*}(t)-F^{*}\left(x^{(2)}(t)\right)+F^{*}(1 / 2)\right] .
$$

By equation (11) it is an increasing function (on $\left[0, t_{1}\right] \cup\left[t_{2}, t_{3}\right]$ it coinsides with $\left.y^{(1)}\right)$. By monotonicity of the functions in equation (10), we get $y^{(3, T)}(t) \geq y^{(1)}(t)$ on $\left[t_{1}, t_{2}\right]$ and $y^{(3, T)}(t) \leq y^{(1)}(t)$ on $\left[t_{3}, 1\right]$. Since $y^{(3, T)}$ is continuous, the intervals on which it has to be corrected are strictly inside the intervals on which we have corrected $x^{(2, T)}$. In Figure 6 the temporary $y^{(3, T)}(t)$ is already optimal (for given 


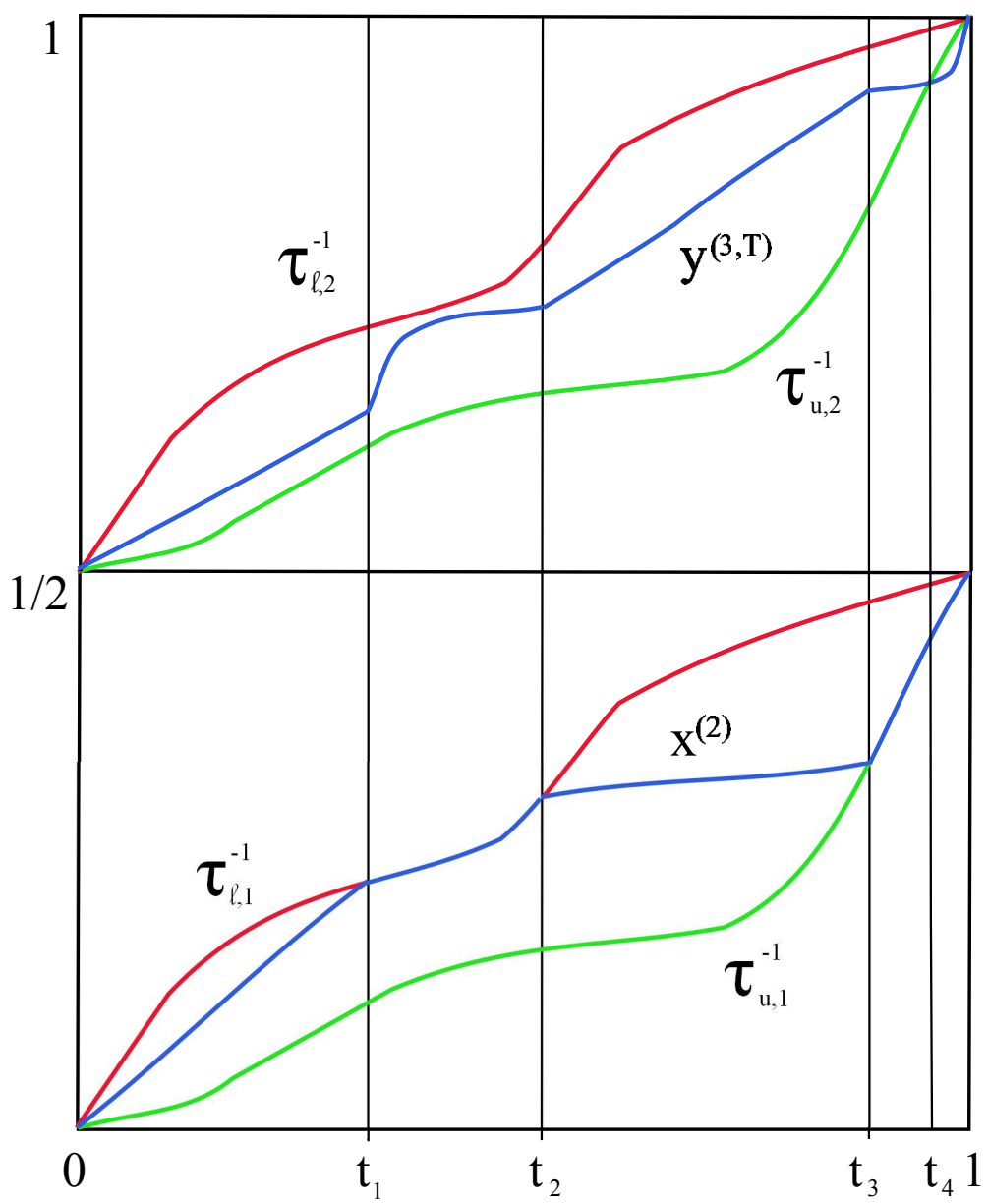

Figure 6 . The inverse $x^{(2)}(t)$ and the corresponding temporary inverse $y^{(3, T)}(t)$.

$\left.x^{(2)}(t)\right)$ on interval $\left[0, t_{4}\right]$ and has to be corrected on $\left[t_{4}, 1\right]$. We set

$$
y_{3}(t)=\max \left\{y^{(3, T)}(t), \tau_{u, 2}^{-1}(t)\right\} .
$$

Again, it can be shown the same way as in Proposition 5 that, for given $x^{(2)}$, this choice of $y^{(3)}$ produces the minimal possible value of $J_{1}\left(\boldsymbol{\eta}^{(2)}\right)$, where

$$
\boldsymbol{\eta}^{(2)}(\theta)= \begin{cases}\left(x^{(2)}\right)^{-1}(\theta), & 0 \leq \theta \leq 1 / 2 \\ \left(y^{(3)}\right)^{-1}(\theta), & 1 / 2<\theta \leq 1 .\end{cases}
$$

In particular, $J_{1}\left(\boldsymbol{\eta}^{(2)}\right) \leq J_{1}\left(\boldsymbol{\eta}^{(1)}\right)$. The map $y^{(3)}$ is shown in Figure 7 .

The first loop of the algorithm is complete. Now we iterate, producing the sequence of selectors $\boldsymbol{\eta}^{(n)}$. For odd indices $n$, we have

$$
\boldsymbol{\eta}^{(n)}(\theta)=\left(x^{(n+1)}\right)^{-1}(\theta) \cdot \chi_{[0,1 / 2]}(\theta)+\left(y^{(n)}\right)^{-1}(\theta) \cdot \chi_{[1 / 2,1]}(\theta),
$$

and for even indices $n$, we have

$$
\boldsymbol{\eta}^{(n)}(\theta)=\left(x^{(n)}\right)^{-1}(\theta) \cdot \chi_{[0,1 / 2]}(\theta)+\left(y^{(n+1)}\right)^{-1}(\theta) \cdot \chi_{[1 / 2,1]}(\theta) .
$$




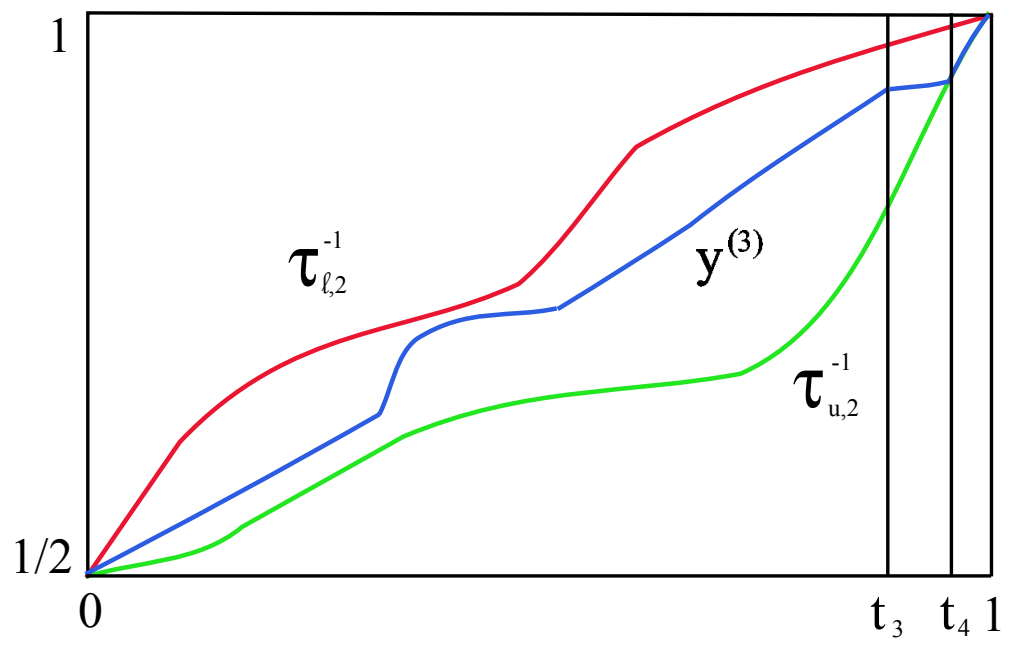

Figure 7 . The inverse $y^{(3)}(t)$.

The values $J_{1}\left(\boldsymbol{\eta}^{(n)}\right)$ are decreasing.

The following propositions justify the validity of the steps in the algorithm.

Proposition 9. Let $x(t)$ and $y(t)$ satisfy equation (10). If $y(t)$ is increasing, $y^{\prime}(t)<1, y(t) \geq t$ and $y(t) \geq 1 / 2$, then $x(t)$ is increasing, $x^{\prime}(t)<1, x(t) \leq t$ and $x(t) \leq 1 / 2$ for $0 \leq t \leq 1$.

Proof. Differentiating (10), we obtain

$$
F^{* \prime}=F^{*^{\prime}}(x) \cdot x^{\prime}+F^{*^{\prime}}(y) \cdot y^{\prime},
$$

or

$$
x^{\prime}(t)=\frac{F^{* \prime}(t)-F^{* \prime}(y(t)) \cdot y^{\prime}(t)}{F^{*^{\prime}}(x(t))} .
$$

We assume that $F^{* \prime}$ is decreasing. Since $y^{\prime}<1$, we have

$$
x^{\prime}(t)>\frac{F^{*^{\prime}}(t)-F^{*^{\prime}}(y(t))}{F^{*^{\prime}}(x(t))},
$$

and since $y(t)>t$ we have $F^{* \prime}(t)>F^{* \prime}(y(t))$ and thus

$$
x^{\prime}>0 .
$$

We now show that $x(t) \leq t$. Our assumption is that $y(t)$ is increasing and $y(t)>$ $1 / 2$. Since $F^{*}(t)=F^{*}(x(t))+F^{*}(y(t))-F^{*}(1 / 2)$, it follows that $F^{*}(t)>F^{*}(x(t))$. Since $F^{*}$ is an increasing function, we have $x(t)<t$.

We rewrite (10) in the form

$$
F^{*}(t)-F^{*}(y(t))=F^{*}(x(t))-F^{*}(1 / 2) .
$$

Since $F^{*}$ is an increasing function and $y(t) \geq t$, we have $x(t)<1 / 2$.

Now we show that $x^{\prime}<1$. Since $F^{*^{\prime}}(y(t)) \cdot y^{\prime}(t)>0$, we have

$$
x^{\prime}(t)<\frac{F^{*^{\prime}}(t)}{F^{*^{\prime}}(x(t))} \leq 1,
$$

since $x(t) \leq t$. 
Proposition 10. Let $x(t)$ and $y(t)$ satisfy equation (10). Assume that $x(t)$ is increasing, $x^{\prime}(t)<1, x(t) \leq t$ and $x(t) \leq 1 / 2$. If $y(t)$ is well defined, then $y(t) \geq t$ and $y(t) \geq 1 / 2$ for $0 \leq t \leq 1$.

Proof. We will show that $y(t) \geq t$ and $y(t)>1 / 2$. We rewrite equation (10) in the form

$$
F^{*}(t)-F^{*}(y(t))=F^{*}(x(t))-F^{*}(1 / 2) .
$$

Since $x(t) \leq 1 / 2$ and $F^{*}$ is increasing we have $y(t) \geq t$. By rewriting (10) in the form

$$
F^{*}(t)-F^{*}(x(t))=F^{*}(y(t))-F^{*}(1 / 2)
$$

and noting that $x(t) \leq t$ and $F^{*}$ is increasing, we obtain $y(t)>1 / 2$.

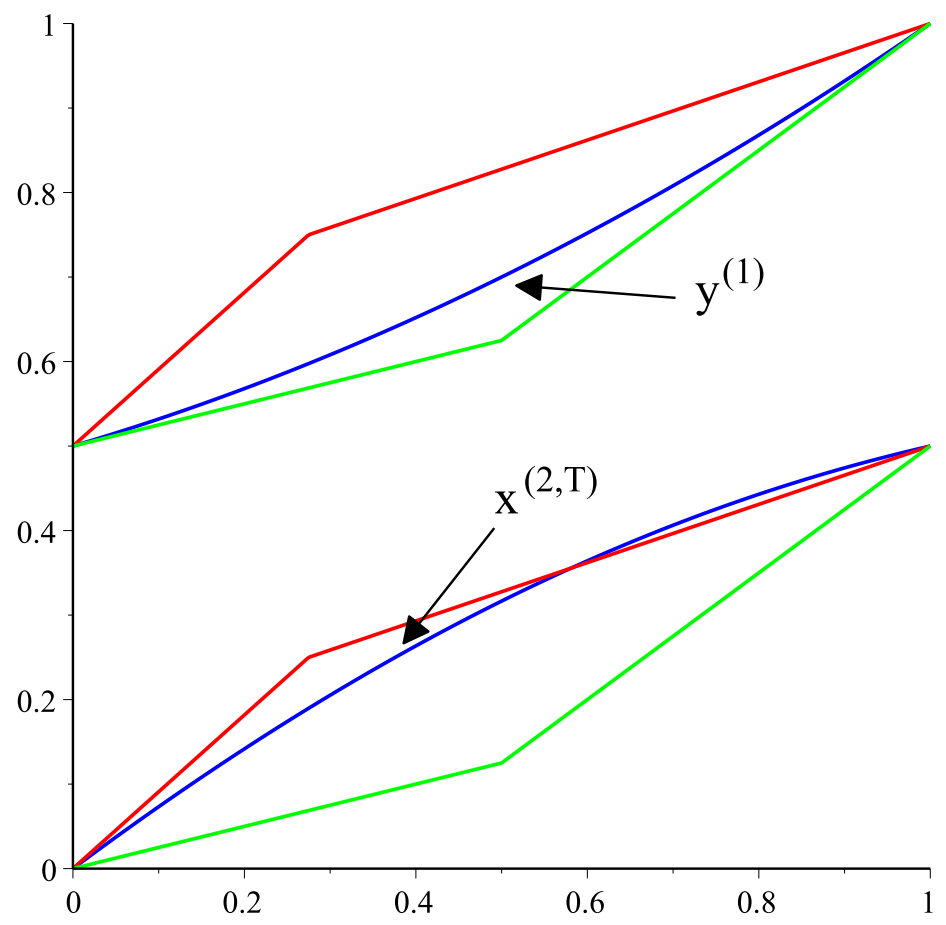

Figure 8. Example 11: The inverse branches of $\tau_{\ell}, \tau_{u}$ (red and green), the initial inverse $y^{(1)}$ and the corresponding temporary $x^{(2, T)}$ (blue).

\section{Example 11.}

We consider the boundary maps

$$
\begin{aligned}
\tau_{\ell}(t)=(1.1 t) \chi_{[0,1 / 4]}(t)+(2.9 t & -0.45) \chi_{[1 / 4,1 / 2]}(t) \\
& +(1.1 t-0.55) \chi_{[1 / 2,3 / 4]}(t)+(2.9 t-1.9) \chi_{[3 / 4,1]}(t),
\end{aligned}
$$




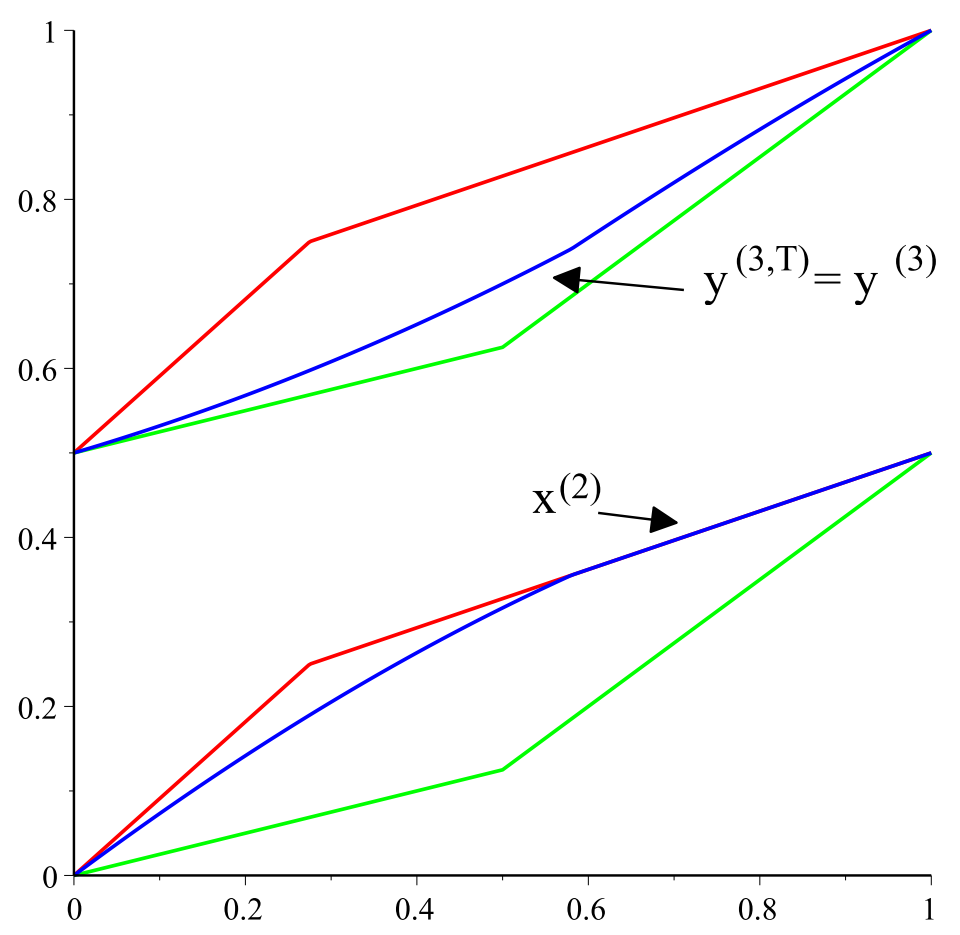

Figure 9. Example 11: The inverse branches of $\tau_{\ell}, \tau_{u}$ (red and green), the corrected map $x^{(2)}$ and the corresponding inverse $y^{(3, T)}=y^{(3)}$ (blue).

and

$$
\begin{aligned}
\tau_{u}(t)=(4 t) \chi_{[0,1 / 8]}(t)+\frac{1}{3}(4 t+ & 1) \chi_{[1 / 8,1 / 2]}(t) \\
& +(4 t-2) \chi_{[1 / 2,5 / 8]}(t)+\frac{1}{3}(4 t-1) \chi_{[5 / 8,1]}(t),
\end{aligned}
$$

and the concave target distribution function $F^{*}(t)=(9 / 8) t-(1 / 8) t^{2}$. The graphs of the inverse branches of the maps $\tau_{\ell}, \tau_{u}$ are shown in Figures 8,9 (red and green). It can be checked that condition (ii) is satisfied. We start with $y^{(1)}(t)=t^{2} / 5+3 t / 10+$ $1 / 2$ shown in Figure 8 together with the corresponding temporary inverse $x^{(2, T)}(t)$. The corrected map $x^{(2)}(t)$ is shown in Figure 9 together with the corresponding inverse $y^{(3, T)}(t)$. Since $y^{(3, T)}(t)$ is admissible, we set $y^{(3)}(t)=y^{(3, T)}(t)$ and the procedure is complete. The selector $\boldsymbol{\eta}^{(2)}$ whose inverse branches are $x^{(2)}$ and $y^{(3)}$ preserves the distribution function $F^{*}$. We have $J_{1}\left(\boldsymbol{\eta}^{(2)}\right)=0$.

Example 11 continued: Since the maps $\tau_{\ell}, \tau_{u}$ in Example 11 are symmetric we can look for an optimal selector using this symmetry (part B of Section 4). The equation for $x$ is

$$
F^{*}(t)=F^{*}(x)+F^{*}(x+1 / 2)-F^{*}(1 / 2)
$$




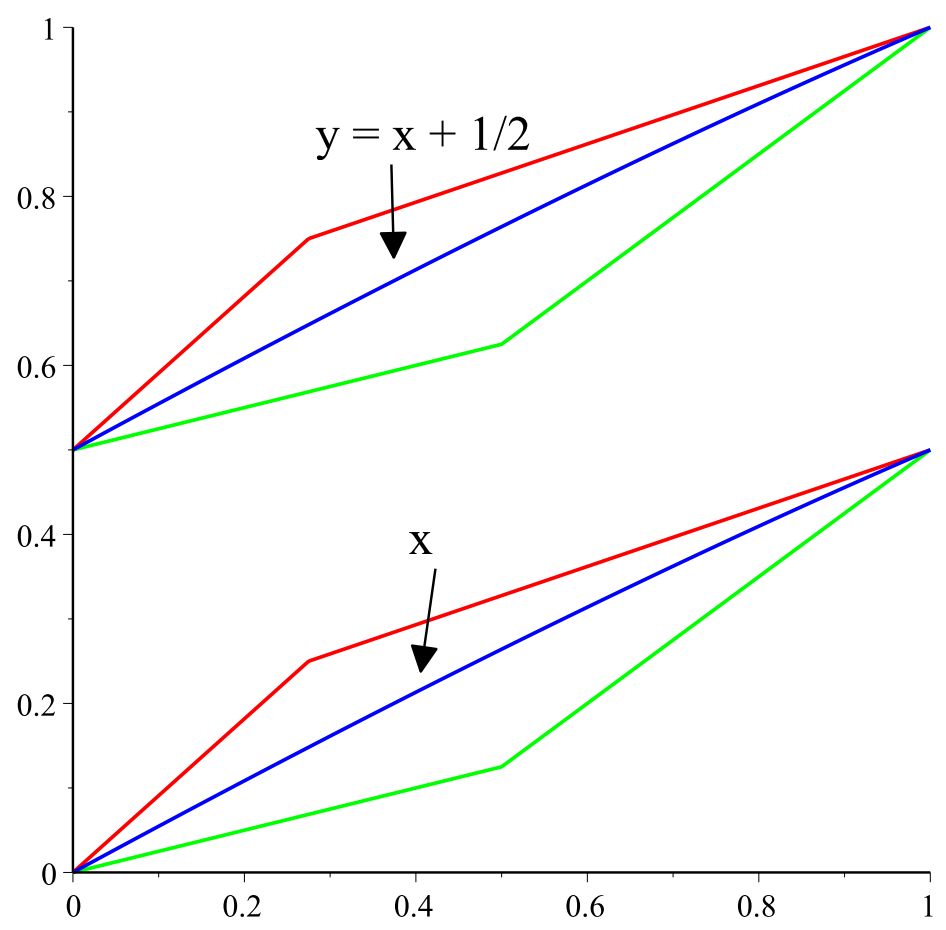

Figure 10. Example 11 continued: The inverse branches of $\tau_{\ell}, \tau_{u}$ (red and green) and the symmetric inverse branches of $\boldsymbol{\eta}: x$ and $y=x+1 / 2$ (blue).

The solution is $x(t)=2.472222222-0.02777777778 \sqrt{648 t^{2}-3528 t+7921}$ and we set $y(t)=x(t)+1 / 2$. It turns out that the function $\boldsymbol{\eta}$ whose inverse branches are these $x(t)$ and $y(t)$ is a selector. See Figure 10. The selector $\boldsymbol{\eta}$ preserves the distribution function $F^{*}$ and we have $J_{1}(\boldsymbol{\eta})=0$. Apparently this solution is different from the solution obtained in the previous example. This shows that the optimalization problem does not have a unique solution. 


\section{REFERENCES}

[1] Antosievicz, A. and Cellina, A., Continuous selectors and differential relations, Jour. Diff. Equations, 19, 386-398, 1975.

[2] Aubin, Jean-Pierre; Frankowska, Helene Set-valued analysis. Systems \& Control: Foundations \& Applications, 2. Birkhauser Boston,Inc., Boston, MA, 1990.

[3] A. Boyarsky and P. Góra, Laws of Chaos. Invariant Measures and Dynamical Systems in One Dimension, Probability and its Applications, Birkhäuser, Boston, MA, 1997.

[4] A. Boyarsky, P. Góra and W. Bahsoun, Folding maps and functional equations, Dynamical Systems, an International Journal 21, no. 2 (2006), 235-43.

[5] Debreu, G., Theory of Value, John Wiley, New York, 1959.

[6] Ershov, S. V., Malinetskii, G. G., Solution of an inverse problem for the Perron-Frobenius equation,) Zh. Vychisl. Mat. i Mat. Fiz. 28 (1988), no. 10, 1491-1497, 1598; translation in U.S.S.R. Comput. Math. and Math. Phys. 28 (1988), no. 5, 136-141 (1990).

[7] P. Góra, A. Boyarsky and Z. Li, Selections and absolutely continuous invariant measures, Journal of Mathematical Analysis and Applications, 413(2014), 100-113.

[8] P. Góra and A. Boyarsky, Absolutely continuous invariant measures for random maps with position dependent probabilities, J. Math. Anal. Appl. 278 (2003), 225-242.

[9] Góra, P., Boyarsky, A., A matrix solution to the inverse Perron-Frobenius problem, Proc. Amer. Math. Soc. 118 (1993), no. 2, 409-414.

[10] P. Góra, Z. Li, A. Boyarsky and H. Proppe, Toward a mathematical holographic principle, Journal of Stat. Phys. 156, Issue 4, 2014, 775-799.

[11] Hildebrand, G., Core and Equilibria of a Large Economy, Princeton University Press, 1974.

[12] Kaczyński, Tomasz, Multivalued maps as a tool in modeling and rigorous numerics, J. Fixed Point Theory Appl. 4, (2008), no. 2,151-176.

[13] B. MacCluer, Elementary Functional Analysis, Graduate Text in Mathematics, Vol. 253, Springer, New York, 2009.

[14] Michael, Continuous selections I, Annals of Mathematics, Vol. 63, No.2, 361-382, 1956. Continuous Selections II, Annals of Mathematics, Vol. 65, No. 2, 375-390, 1957.

[15] Rogers, Alan, Shorten, Robert, Heffernan, Daniel M., A novel matrix approach for controlling the invariant densities of chaotic maps, Chaos Solitons Fractals 35 (2008), no. 1, 161-175.

[16] Samet, D, Continuous selections for vector measures, Mathematics of Operations Research, Vol. 12, No. 3, 536-643, , 1987.

[17] Sanchez-Gabites, J.J. and Sanjurjo, J.M.R, Multivalued maps, selections and dynamical systems, Topology and its Applications, 155, 874-882, 2008.

[18] Santos, M.S. and Peralta-Alva, A, Ergodic invariant measures for non-optimal dynamic economies, preprint, May 12, 2013.

[19] Swishchuk, A. and Islam, Sh., Random Dynamical Systems in Finance, CRC Press, Boca Raton, 2013.

[20] Tolstonogov, A. A., Extreme continuous selectors of multivalued maps and their applications, Jour. Diff. Equations, 122 (1995), 161-180.

[21] Tolstonogov, A. A. and Tolstonogov, D. A., $L_{p}$-continuous extreme selectors of multifuncitons with decomposable values: existence theorems, Set-Valued Analysis, 4 (1996), 173-203.

(A. Boyarsky) Department of Mathematics and Statistics, Concordia University, 1455 de Maisonneuve Blvd. West, Montreal, Quebec H3G 1M8, Canada

E-mail address, A. Boyarsky: abraham.boyarsky@concordia.ca

(P. Góra) Department of Mathematics and Statistics, Concordia University, 1455 de Maisonneuve Blvd. West, Montreal, Quebec H3G 1M8, Canada

E-mail address, P. Góra: pawel.gora@concordia.ca

(Z. Li) Department of Mathematics, Honghe University, Mengzi, Yunnan 661100, China E-mail address, Z. Li: zhenyangemail@gmail.com 